J. Dairy Sci. 99:7033-7042

http://dx.doi.org/10.3168/jds.2016-11074

(C) 2016, THE AUTHORS. Published by FASS and Elsevier Inc. on behalf

of the American Dairy Science Association ${ }^{\circledR}$. This is an open access article under

the CC BY-NC-ND license (http://creativecommons.org/licenses/by-nc-nd/3.0/).

\title{
Evaluation of different methods to detect microbial hygiene indicators relevant in the dairy industry
}

\author{
C. J. Hervert, A. S. Alles, N. H. Martin, K. J. Boor, and M. Wiedmann ${ }^{1}$ \\ Milk Quality Improvement Program, Department of Food Science, Cornell University, Ithaca, NY 14853
}

\begin{abstract}
It is estimated that $19 \%$ of the total food loss from retail, food service, and households comes from dairy products. A portion of this loss may be attributed to premature spoilage of products due to lapses in sanitation and postpasteurization contamination at the processing level. Bacterial groups including coliforms, Enterobacteriaceae (EB), and total gram-negative organisms represent indicators of poor sanitation or postpasteurization contamination in dairy products worldwide. Although Petrifilms (3M, St. Paul, MN) and traditional selective media are commonly used for the testing of these indicator organism groups throughout the US dairy industry, new rapid methods are also being developed. This project was designed to evaluate the ability of different methods to detect coliforms, EB, and other gram-negative organisms isolated from various dairy products and dairy processing environments. Using the Food Microbe Tracker database, a collection of 211 coliform, EB, and gram-negative bacterial isolates representing 25 genera associated with dairy products was assembled for this study. We tested the selected isolates in pure culture (at levels of approximately 15 to 300 cells/test) to evaluate the ability of $3 \mathrm{M}$ Coliform Petrifilm to detect coliforms, 3M Enterobacteriaceae Petrifilm, violet red bile glucose agar, and an alternative flow cytometry-based method (bioMérieux D-Count, Marcy-l'Étoile, France) to detect EB, and crystal violet tetrazolium agar to detect total gramnegative organisms. Of the 211 gram-negative isolates tested, $82 \%(174 / 211)$ had characteristic growth on crystal violet tetrazolium agar. Within this set of 211 gram-negative organisms, 175 isolates representing 19 EB genera were screened for detection using EB selec-
\end{abstract}

Received February 22, 2016.

Accepted May 29, 2016.

${ }^{1}$ Corresponding author: mw16@cornell.edu tive/differential testing methods. We observed positive results for $96 \%(168 / 175), 90 \%(158 / 175)$, and $86 \%$ $(151 / 175)$ of EB isolates when tested on EB Petrifilm, violet red bile glucose agar, and D-Count, respectively; optimization of the cut-off thresholds for the D-Count may further improve its sensitivity and specificity, but will require additional data and may vary in food matrices. Additionally, 74\% (129/175) of the EB isolates tested positive as coliforms. The data obtained from this study identify differences in detection between 5 microbial hygiene indicator tests and highlight the benefits of EB and total gram-negative testing methods.

Key words: coliform, Enterobacteriaceae, gramnegative, indicator organism

\section{INTRODUCTION}

Since 1914, the United States has used coliform organisms to indicate the microbiological quality and safety of drinking water (US Treasury Department, 1914). Over the course of the next $100 \mathrm{yr}$, the use of coliforms as indicator organisms expanded, becoming the standard hygienic quality test for many food and beverage products. The dairy industry has long since used coliforms for this purpose as they are represented in over 20 genera of gram-negative, non-spore-forming rods, which lack the capability to survive typical milk heat treatments (e.g., HTST pasteurization) and can hence act as indicators of postpasteurization contamination (Imhoff, 2005; Masiello et al., 2016). The phenotypic characteristic that defines coliform bacteria is their ability to ferment lactose, resulting in gas and acid production within $48 \mathrm{~h}$ at $35^{\circ} \mathrm{C}$ (Feng et al., 2002). It is this property that distinguishes coliform organisms from other lactose nonfermenters (e.g., Pseudomonas sp.) when plated on selective and differential coliform media. Strict FDA requirements regarding coliform and total bacterial limits have been put in place to ensure minimum standards are met for the hygienic quality of dairy foods. These standards are outlined in the 2011 
Pasteurized Milk Ordinance and require coliform counts in grade A pasteurized milk to not exceed $10 \mathrm{cfu} / \mathrm{mL}$ (FDA, 2011). In addition to coliforms being indicative of the hygienic status of dairy products and processing environments, they have been shown to have implications on the sensory quality of dairy products. Past studies demonstrate that select strains from common coliform genera grow at refrigeration temperatures and exhibit proteolytic and lipolytic capabilities (Wessels et al., 1989; Masiello et al., 2016). The production of proteolytic and lipolytic enzymes may have an influence on the consumer acceptance of dairy products, as pasteurized milk samples contaminated with coliforms are associated with significant decreases in sensory scores on d 14 of shelf life when compared with uncontaminated samples (Martin et al., 2012). These instances of postpasteurization contamination with spoilage microorganisms may contribute to the dairy product food loss observed at the retail, food service, and household levels (Gunders, 2012).

Despite the longstanding use of coliforms as hygiene indicators in the US dairy industry, recent work indicates that coliforms represent less than $50 \%$ of the bacterial contaminants involved in postpasteurization contamination of fluid milk (Ranieri and Boor, 2009). An alternative group of indicators used widely across Europe are organisms within the taxonomic family Enterobacteriaceae (EB; European Communities Regulation, 2010). This group of organisms is composed of gram-negative, heat-labile, glucose fermenters and represents a broad range of dairy-related genera with the potential to indicate postpasteurization contamination. With the notable exception of specific strains of lactose-fermenting Aeromonas (Abbott et al., 2003), the EB group also encompasses classic coliform genera (Imhoff, 2005). Typical media for the enumeration of EB include violet red bile glucose agar (VRBGA) and EB Petrifilm, though new methods for EB detection are also being developed.

Although the EB group provides a more encompassing range of hygiene indicators when compared with coliforms, several the gram-negative, postpasteurization contaminants found in fluid milk (e.g., Pseudomonas) do not fall into this group. Prior studies indicate that Pseudomonas spp. are dominant among gram-negative organisms isolated from pasteurized milk (Ranieri and Boor, 2009) and generate unsatisfactory sensory defects through the production of proteases and lipases (Sørhaug and Stepaniak, 1997; Hayes et al., 2002). Subsequent to postpasteurization contamination, the growth of Pseudomonas and other non-EB gram-negative organisms at refrigeration temperatures has been shown to be indicative of the shelf life and overall consumer acceptance of milk (Dogan and Boor, 2003). Additionally, a recent study highlighted the unique spoilage potential of certain biovars of pigment-producing Pseudomonas isolated from fresh, low-acid cheese (Martin et al., 2011). It is for this reason that the "blanket-like" approach of screening for total gram-negative organisms offers a more comprehensive indicator of postpasteurization contamination, sanitation quality, and dairy shelf life when compared with other indicator organism groups. Crystal violet tetrazolium agar (CVTA) is the standard method for enumerating gram-negative organisms including Pseudomonas in dairy products (Frank and Yousef, 2004), while inhibiting gram-positive growth through the inclusion of crystal violet.

The objective of this study was to screen a diverse collection of dairy-relevant coliform, EB, and general gram-negative organisms for detection on Coliform Petrifilm, EB Petrifilm, VRBGA, CVTA, as well as by an alternative flow cytometry-based method. The resulting data provide new information on potential use of these indicator organism groups in the dairy industry and identify optimal detection methods for different indicator organism groups and gram-negative genera.

\section{MATERIALS AND METHODS}

\section{Isolate Selection}

Through utilization of the Food Microbe Tracker database (www.foodmicrobetracker.com; Vangay et al., 2013), a collection of 211 gram-negative bacterial isolates representing a broad range of organisms commonly associated with dairy products and processing environments was assembled for the purpose of this study. Isolation sources included pasteurized milk (117/211), dairy processing plant environment/dairy food product (42/211), raw milk $(16 / 211)$, cheese $(11 / 211)$, environment/food $(7 / 211)$, unspecified $(6 / 211)$, infant formula $(6 / 211)$, laboratory heat-treated raw milk $(3 / 211)$, pasteurized chocolate milk (2/211), and clinical (1/211; Supplemental Table S1; http://dx.doi.org/10.3168/ jds.2016-11074). Within the collection, 175 isolates from 19 genera were classified as falling into the EB family, whereas 36 isolates from 6 genera were classified as non-EB, gram-negative. Genus identification information for isolates was obtained through the Food Microbe Tracker database based on previously performed partial 16S DNA sequencing, as described in prior studies (Huck et al., 2007). Additionally, 50\% $(106 / 211)$ of the isolates were previously described in one or more studies (Marie Yeung et al., 2003; Ranieri and Boor, 2009; Martin et al., 2011; Van Tassell et al., 2012; Ivy et al., 2013; Masiello et al., 2016). 


\section{Enumeration, Preparation, and Testing of Pure Cultures}

Prior to undergoing selective and differential testing, the selected isolates were first streaked from frozen culture onto brain heart infusion (BHI) agar (Becton Dickinson, Sparks, MD) and incubated for $24 \mathrm{~h}$ at 31 to $33^{\circ} \mathrm{C}$. An isolated colony was selected for each isolate and used to inoculate a tube containing $5 \mathrm{~mL}$ of BHI broth (Becton Dickinson), followed by $18 \mathrm{~h}$ of incubation at 32 $\pm 1^{\circ} \mathrm{C}$. Incubated broths were diluted with phosphate buffer by a factor of 1,000 before spiral plating onto BHI agar using an automated spiral plater (Advanced Instruments Inc., Norwood, MA). Following $24 \mathrm{~h}$ of incubation at $32 \pm 1^{\circ} \mathrm{C}$, colony growth on the plates was enumerated on a Color Q-Count instrument (Advanced Instruments Inc.) to obtain count data on the $18 \mathrm{~h}$ BHI broth. A new set of BHI broths was then inoculated using the same isolated colonies that had been used for the initial experiments, followed by incubation for $18 \mathrm{~h}$ at $32 \pm 1^{\circ} \mathrm{C}$. The count data were used to create serial dilutions of the new broths resulting in countable levels when plated on the various medium types tested. These medium types included CVTA (Frank and Yousef, 2004), VRBGA (Becton Dickinson, Sparks, MD), 3M Coliform Petrifilm (3M, St. Paul, MN), 3M Enterobacteriaceae Petrifilm (3M), as well as BHI agar to serve as a control medium on which all isolates were expected to grow. Dilutions were plated on CVTA, VRBGA, and $\mathrm{BHI}$ agar using the nonexponential $100 \mu \mathrm{L}$ mode of the automated spiral plater resulting in colony counts of approximately 15 to $300 \mathrm{cfu} /$ plate for most isolates. To obtain comparable colony-forming unit counts to those that were obtained using the automated spiral plater, $1 \mathrm{~mL}$ of a 10-fold further dilution was plated onto both the Coliform and EB Petrifilms for each respective isolate. Upon completion of plating, all VRBGA plates, BHI plates, Coliform Petrifilms, and EB Petrifilms were incubated aerobically for $24 \mathrm{~h}$ at $32 \pm 1^{\circ} \mathrm{C}$ and CVTA plates were incubated aerobically for $48 \mathrm{~h}$ at $21^{\circ} \mathrm{C}$ (Frank and Yousef, 2004). In addition to plating on traditional detection medium, isolates were tested using a flow cytometry method (D-Count, bioMérieux, Marcy-l'Étoile, France) with a protocol designed to detect EB organisms. The "Presence/Absence Test of EB in Fermented Milk Products Containing Bifidobacterium" application of the D-Count was used to test isolates according to the manufacturer's protocols and procedures; this procedure includes an enrichment step, followed by flow cytometry to allow for EB detection in approximately $13 \mathrm{~h}$ rather than $24 \mathrm{~h}$ for EB Petrifilm and VRBGA. Briefly, for each test isolate, $1 \mathrm{~mL}$ of the serial dilution plated on Coliform and EB Petrifilms was inoculated into $9 \mathrm{~mL}$ of a proprietary EB selective broth. Inoculated broths were incubated for $13 \mathrm{~h}$ at 37 $\pm 1^{\circ} \mathrm{C}$ before testing on the flow cytometry instrument. To test, $10 \mu \mathrm{L}$ of enrichment broth was treated with reagents that label viable EB cells. The sample was then automatically injected into the flow cell analyzer of the D-Count forming a narrow and laminar flow stream. Detectors within the analyzer counted the labeled cells, outputting a value in counts per milliliter of analyzed sample.

\section{Interpretation of Media}

Following the incubation of plates and Petrifilms, colony growth was enumerated visually for Petrifilms, and with the aid of a Color Q-Count instrument for plates. Differential medium types were also examined for typical or atypical growth characteristics indicating a positive or negative result for their respective differential capabilities. A positive result on EB Petrifilm was triggered through acid, gas, or gas and acid production generated from the fermentation of glucose. Per the manufacturer's instructions, these characteristics result in red colonies with yellow zones for acid-producing isolates, red colonies with associated gas bubbles for gas-producing isolates, and red colonies with yellow zones and associated gas bubbles for acid and gas-producing isolates. The EB glucose fermenters produce red colonies on VRBGA with red-purple halos (bile precipitation) in the presence of neutral red, a $\mathrm{pH}$ indicator. Isolates demonstrating lactose fermentation were classified as falling into the coliform group and were identified through formation of red colonies with associated gas bubbles when plated on Coliform Petrifilm, as outlined in the manufacturer's instructions. Characteristic growth of gram-negative isolates was exhibited through the presence of dark red colony formation when plated on CVTA (Frank and Yousef, 2004). For the D-Count, a positive result for an EB organism was defined as having greater than 100 counts/ $\mathrm{mL}$ upon completion of the test.

\section{Data Analyses}

All data were managed using Excel (version 14.5.4, Microsoft, Redmond, WA) and all linear models were created in RStudio (version 0.98.149, RStudio Inc., Boston, MA). Isolates that showed no growth on a given selective and differential media (i.e., 0 cfu/plate) were not included in the calculation of slope and $\mathrm{R}^{2}$ for the linear models. Sensitivity, or the true positive rate, is defined as the proportion of true positives that 
Table 1. Detection of the 211 isolates of the study using select methods with reference to test sensitivity, specificity, and \% coliforms detected

\begin{tabular}{|c|c|c|c|c|c|}
\hline \multirow[b]{2}{*}{ Detection method } & \multicolumn{2}{|c|}{ Detection of } & \multirow[b]{2}{*}{$\begin{array}{c}\text { Sensitivity } \\
(\%)\end{array}$} & \multirow[b]{2}{*}{$\begin{array}{l}\text { Specificity } \\
(\%)\end{array}$} & \multirow[b]{2}{*}{$\begin{array}{c}\text { Coliforms } \\
\text { detected }^{1}(\%)\end{array}$} \\
\hline & $\begin{array}{c}\mathrm{EB} \\
(\mathrm{n}=175)\end{array}$ & $\begin{array}{l}\text { Non-EB } \\
(\mathrm{n}=36)\end{array}$ & & & \\
\hline Brain heart infusion (BHI) agar & 175 & 36 & $100^{2}$ & $\mathrm{NA}^{3}$ & 100 \\
\hline Crystal violet tetrazolium (CVTA) agar & 155 & 19 & $82^{2}$ & NA & 97 \\
\hline Violet red bile glucose agar & 158 & 3 & $90^{4}$ & $92^{5}$ & 91 \\
\hline Enterobacteriaceae (EB) Petrifilm ${ }^{6}$ & 168 & 4 & $96^{4}$ & $89^{5}$ & 100 \\
\hline D-Count & 151 & 0 & $86^{4}$ & $100^{5}$ & 93 \\
\hline \multicolumn{6}{|c|}{$\begin{array}{l}{ }^{1} \text { Proportion of coliform isolates detected is based on the } 129 \text { coliform isolates included in the isolate set used. } \\
{ }^{2} \text { Sensitivity calculations for BHI and CVTA were based on all } 211 \text { gram-negative isolates tested. } \\
{ }^{3} \mathrm{NA}=\text { not applicable. } \\
{ }^{4} \text { Sensitivity calculations for the EB specific assays were based on the } 175 \mathrm{~EB} \text { isolates included in the isolate } \\
\text { set used. } \\
{ }^{5} \text { Specificity calculations for the EB specific assays were based on the } 36 \text { non-EB isolates included in the isolate } \\
\text { set used. }\end{array}$} \\
\hline
\end{tabular}

are correctly identified as such. Specificity, or the true negative rate, is defined as the proportion of true negatives that are correctly identified as such. The proportion of overall agreement $\left(\mathbf{P}_{\mathbf{o}}\right)$ is the proportion of cases for which both testing methods agree and is calculated as follows:

$$
=\frac{\begin{array}{l}
\text { Positive Result Agreements }+ \\
\text { Negative Result Agreements }
\end{array}}{\text { Total Tests Performed }} .
$$

\section{RESULTS}

\section{Detection Methods Differed in Sensitivity and Specificity for Their Respective Target Organisms}

In this study, we evaluated testing methods for the detection of 3 dairy hygiene indicator organism groups including (1) Coliform Petrifilm for coliform detection; (2) EB Petrifilm, VRBGA, and the D-Count for EB detection; and (3) CVTA for detection of total gram-negative organisms (see Supplemental Table S1, http://dx.doi.org/10.3168/jds.2016-11074, for detailed results on all 211 isolates tested). Out of the 211 gramnegative organisms, 129 yielded positive results on Coliform Petrifilm and were hence classified as coliforms (Supplemental Table S1). On CVTA, 174 of the 211 gram-negative isolates exhibited growth and characteristic colony morphology after incubation for $48 \mathrm{~h}$ for a sensitivity of $82 \%(174 / 211)$.

Based on data for $175 \mathrm{~EB}$ and 36 non-EB isolates, EB Petrifilm was the most sensitive of the EB specific tests, correctly detecting $96 \%(168 / 175)$ of the EB isolates tested (Table 1). The VRBGA and D-Count were less sensitive, detecting $90 \%(158 / 175)$ and $86 \%$
$(151 / 175)$ of $\mathrm{EB}$ isolates, respectively. On the other hand, the D-Count showed the highest specificity for non-EB organisms $(100 \% ; 36 / 36)$, followed by VRBGA $(92 \% ; 33 / 36)$ and EB Petrifilm $(89 \% ; 32 / 36)$. Out of the $168 \mathrm{~EB}$ isolates that tested positive on EB Petrifilm, no isolates exhibited gas production without acid production (Table 2). Isolates exhibiting both acid and gas production were the most common EB organisms on EB Petrifilm (83\%; 139/168), followed by isolates exhibiting acid, but no gas production (17\%; 29/168; Supplemental Table S1, http://dx.doi.org/10.3168/ jds.2016-11074). It is also important to note that 4 Aeromonas isolates, which are not part of the family Enterobacteriaceae, tested positive on EB Petrifilm, whereas 2 Aeromonas and 1 Acinetobacter isolate tested positive on VRBGA (Supplemental Table S1).

To assess consistency in detection results between the 3 EB testing methods, we calculated the proportion of overall agreement $\left(\mathrm{P}_{\mathrm{o}}\right)$. The D-Count and VRBGA showed the highest proportion of agreement $\left(\mathrm{P}_{\mathrm{o}}=0.93\right)$ with 148 positive result agreements and 14 negative result agreements out of the 175 tests performed (Figure 1). We observed lower proportions of overall agreement for EB Petrifilm and VRBGA $\left(\mathrm{P}_{\mathrm{o}}=0.89\right)$, and D-Count and EB Petrifilm $\left(\mathrm{P}_{\mathrm{o}}=0.85\right)$. The EB isolates that were detected on VRBGA, but not on EB Petrifilm included Buttiauxella $(\mathrm{n}=5)$, whereas EB isolates that were detected on EB Petrifilm, but not VRBGA included Rahnella $(\mathrm{n}=8)$, Serratia $(\mathrm{n}=4)$, Citrobacter $(\mathrm{n}=1)$, Plesiomonas $(\mathrm{n}=1)$, and Raoultella $(\mathrm{n}=1)$. The EB isolates that were detected on VRBGA, but not the D-Count, included Hafnia $(\mathrm{n}=1)$ and Yersinia $(\mathrm{n}=9)$, whereas EB isolates that were detected using the D-Count, but not VRBGA included Citrobacter (n $=1)$, Rahnella $(\mathrm{n}=1)$, and Serratia $(\mathrm{n}=1)$. The EB isolates that were detected using the D-Count, but not 


\begin{tabular}{|c|c|c|c|c|c|c|c|}
\hline & \multicolumn{2}{|c|}{ D-Count } & \multicolumn{2}{|c|}{ VRBGA } & \multicolumn{2}{|c|}{ EB Petrifilm } \\
\hline & & $\begin{array}{c}\text { Positive } \\
\text { Detection }\end{array}$ & $\begin{array}{l}\text { Negative } \\
\text { Detection }\end{array}$ & $\begin{array}{l}\text { Positive } \\
\text { Detection }\end{array}$ & $\begin{array}{l}\text { Negative } \\
\text { Detection }\end{array}$ & $\begin{array}{l}\text { Positive } \\
\text { Detection }\end{array}$ & $\begin{array}{l}\text { Negative } \\
\text { Detection }\end{array}$ \\
\hline \multirow{2}{*}{ BHI Agar } & $\begin{array}{l}\text { Positive } \\
\text { Detection }\end{array}$ & 151 & 24 & 158 & 17 & 168 & 7 \\
\hline & $\begin{array}{l}\text { Negative } \\
\text { Detection }\end{array}$ & 0 & 0 & 0 & 0 & 0 & 0 \\
\hline \multirow{2}{*}{$\begin{array}{c}\text { EB } \\
\text { Petrifilm }\end{array}$} & $\begin{array}{c}\text { Positive } \\
\text { Detection }\end{array}$ & 146 & 22 & 153 & 15 & & \\
\hline & $\begin{array}{l}\text { Negative } \\
\text { Detection }\end{array}$ & 5 & 2 & 5 & 2 & & \\
\hline \multirow{2}{*}{ VRBGA } & $\begin{array}{l}\text { Positive } \\
\text { Detection }\end{array}$ & 148 & 10 & & & & \\
\hline & $\begin{array}{l}\text { Negative } \\
\text { Detection }\end{array}$ & 3 & 14 & & & & \\
\hline
\end{tabular}

Figure 1. Comparison of Enterobacteriaceae (EB) detection results for EB Petrifilm (3M, St. Paul, MN), violet red bile glucose agar (VRBGA), and the D-Count. Brain heart infusion (BHI) agar represents the nonselective medium control upon which all EB $(\mathrm{n}=175)$ isolates exhibited growth.

EB Petrifilm, included Buttiauxella $(\mathrm{n}=5)$, whereas EB isolates that were detected on EB Petrifilm, but not the D-Count included Yersinia $(\mathrm{n}=9)$, Rahnella $(\mathrm{n}=$ $7)$, Serratia $(\mathrm{n}=3)$, Hafnia $(\mathrm{n}=1)$, Plesiomonas $(\mathrm{n}=$ $1)$, and Raoultella $(\mathrm{n}=1)$.

\section{Dairy-Relevant Gram-Negative Indicator Organisms Detected by EB and Total Gram-Negative Tests}

In the previous section, we highlighted the ability of each test to detect its respective indicator organism

Table 2. Isolate growth characteristics on Enterobacteriaceae (EB) and Coliform Petrifilm (3M, St. Paul, MN)

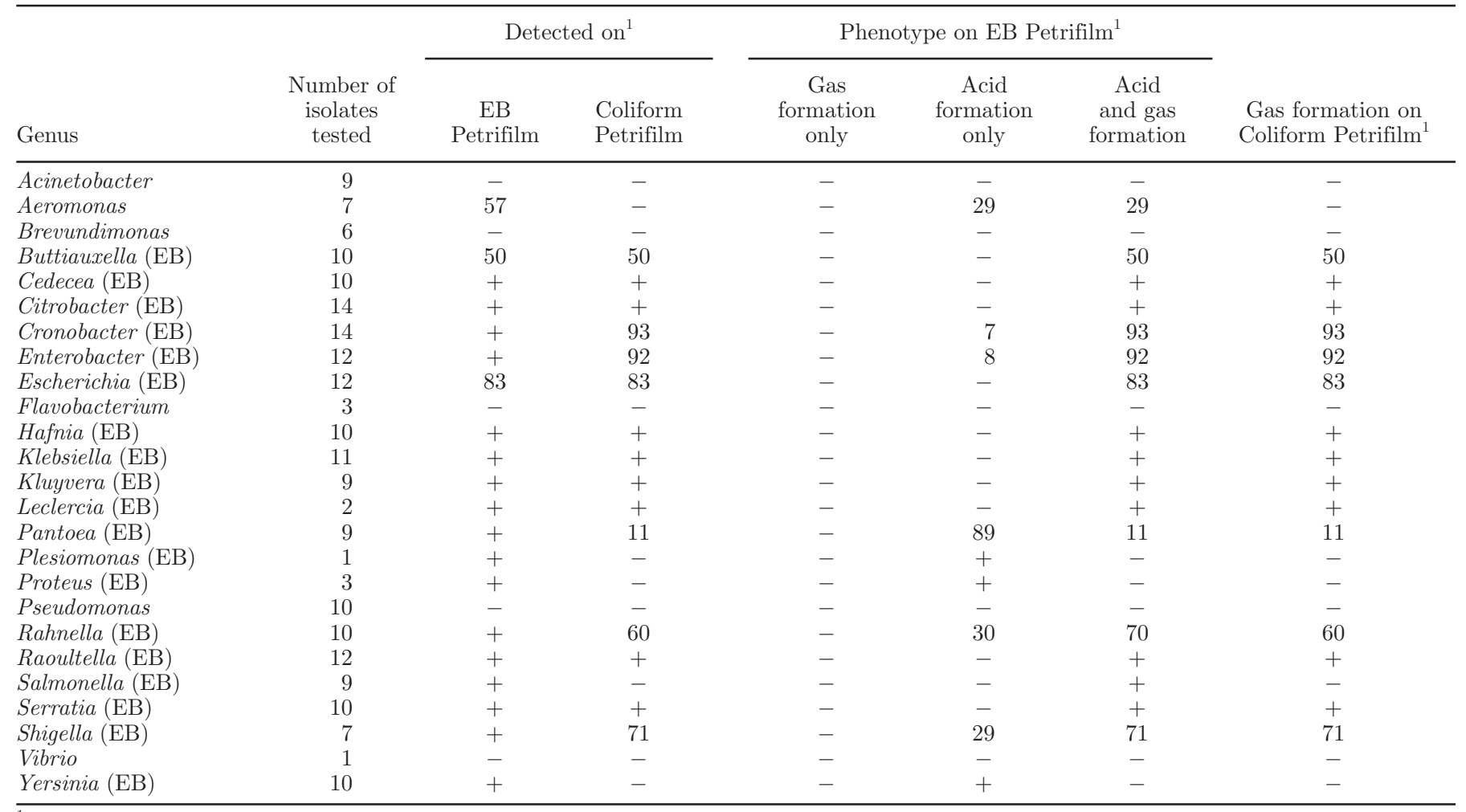

${ }^{1}+$ indicates $100 \%$ of isolates positive; - indicates $100 \%$ of isolates negative; otherwise, number indicates $\%$ of positive isolates. 
groups through calculation of sensitivity and specificity. In addition to these measures of assay performance, we also evaluated the ability of the 5 tests to detect dairy-associated gram-negative bacteria included in our collection of 211 isolates assembled for this study. This is highly relevant as detection of any gram-negative organisms in pasteurized dairy products may be an indication of potential hygiene issues (e.g., postpasteurization contamination) and as several the organisms included in our test set of 211 isolates have been linked to dairy spoilage. Coliform Petrifilm yielded positive results with $61 \%(129 / 211)$ of the organisms tested, representing 15 genera. The 82 organisms that tested negative on Coliform Petrifilm represent a broad range of dairy-associated gram-negative isolates encompassing 17 of the 25 genera tested (Table 2). Among these isolates, 46 were classified as EB organisms [including Salmonella $(\mathrm{n}=9)$ and Yersinia $(\mathrm{n}=10)]$ and 36 were classified as non-EB gram-negative [including Pseudomonas $(\mathrm{n}=10)$ and Acinetobacter $(\mathrm{n}=9)]$.

Consistent with the fact that EB represent a broader range of dairy-associated gram-negative organisms, the 3 EB methods detected a higher proportion of the 211 gram-negative dairy isolates, including typical noncoliform EB genera (e.g., Salmonella, Yersinia), as compared with a coliform test. With 172 positive results, EB Petrifilm detected the highest number of gramnegative organisms of the EB tests $(82 \% ; 172 / 211)$. Of the positive results, 168 were $\mathrm{EB}$ (true positives) and 4 were non-EB (false positives; Table 1). The 39 isolates not detected included 7 EB [false negatives; Buttiauxella $(\mathrm{n}=5)$ and Escherichia $(\mathrm{n}=2)]$ and 32 non-EB gram-negatives (true negatives). The VRBGA detected 161 of the 211 isolates with 158 true positives and 3 false positives (Table 1). The 50 negatives observed on VRBGA consisted of 33 true negatives and 17 false negatives. Notably, Rahnella and Serratia accounted for 12 of the 17 false negatives observed on VRBGA (Supplemental Table S1; http://dx.doi.org/10.3168/ jds.2016-11074). All 151 gram-negative organisms detected using the D-Count were true positives, whereas the 60 undetected isolates consisted of 36 true negatives and 24 false negatives (Table 1). Non-EB gram-negative organisms that were consistently negative across all EB detection methods included Brevundimonas, Flavobacterium, Pseudomonas, and Vibrio.

Despite being the standard method for the detection of dairy-relevant gram-negative organisms, CVTA only detected 2 more gram-negative isolates than EB Petrifilm (174/211). However, CVTA detected several isolates that went undetected by all other detection methods. This included non-EB gram-negative isolates with dairy spoilage significance in the genera Pseu- domonas $(\mathrm{n}=8)$, Aeromonas $(\mathrm{n}=3)$, Acinetobacter $(\mathrm{n}=2)$, and Brevundimonas $(\mathrm{n}=1)$. The 37 gramnegative isolates that CVTA failed to detect included 17 non-EB gram-negative isolates represented in the genera Acinetobacter $(\mathrm{n}=6)$, Brevundimonas $(\mathrm{n}=5)$, Flavobacterium $(\mathrm{n}=3)$, Pseudomonas $(\mathrm{n}=2)$, and Vibrio $(\mathrm{n}=1)$. The remaining 20 isolates came from EB genera including Yersinia $(\mathrm{n}=9)$, Escherichia $(\mathrm{n}=$ $4)$, Shigella $(\mathrm{n}=4)$, Plesiomonas $(\mathrm{n}=1)$, Proteus $(\mathrm{n}=$ $1)$, and Salmonella $(\mathrm{n}=1)$.

\section{EB and Total Gram-Negative Testing Methods Detected Up to $100 \%$ of the Coliforms Represented}

As previously noted, plating on Coliform Petrifilm revealed that 129 organisms from 15 genera possessed the ability to ferment lactose, thus classifying them as coliforms. The more expansive EB and total gramnegative tests were also successful in detecting coliforms with EB Petrifilm detecting 100\% (129/129) of the lactose-fermenting isolates (Table 1). The CVTA, VRBGA, and the D-Count were also highly successful at detecting coliforms, with all methods detecting over $90 \%$ of the 129 coliform isolates tested (Table 1).

All isolates that tested positive as coliforms were classified into EB genera and represented $74 \%(129 / 175)$ of the study's $175 \mathrm{~EB}$ isolates. Coliforms were represented in 15 of the $19 \mathrm{~EB}$ genera, though individual genera differed in the proportion of isolates that were identified as coliforms. From the 15 genera containing coliform organisms, we observed 8 genera where all isolates were characterized as coliforms (e.g., Cedecea and Klebsiella; Table 2). On the other hand, for a total of 4 EB genera (e.g., Salmonella and Yersinia; Table 2), none of the isolates tested as coliforms. Finally, 7 genera included both coliform and noncoliform isolates, including the genus Pantoea (with 1/9 isolates classified as coliforms) and the genus Cronobacter (with 13/14 isolates classified as coliforms; Table 2).

\section{Selective Medium-Based Detection Methods Exhibited a Reduced Recovery of Pure Cultures}

All isolates selected for this study exhibited growth when plated on nonselective BHI agar. To analyze the recovery of the isolates when plated on the selective/ differential medium types, we generated linear models and compared the slopes of the regression lines (Figure 2). A slope of 1 indicates that, on average, the same number of colonies exhibit growth on the nonselective BHI agar as the selective/differential media. Therefore, slope values of $<1$ indicate a lower average recovery of pure cultures grown on selective/differential 
(a.)

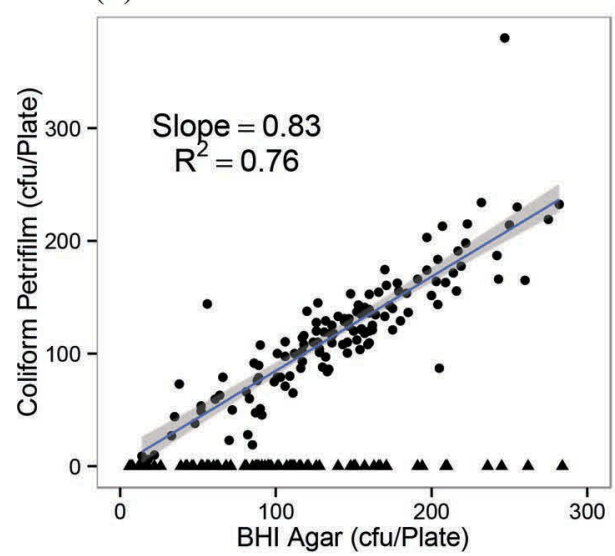

(c.)

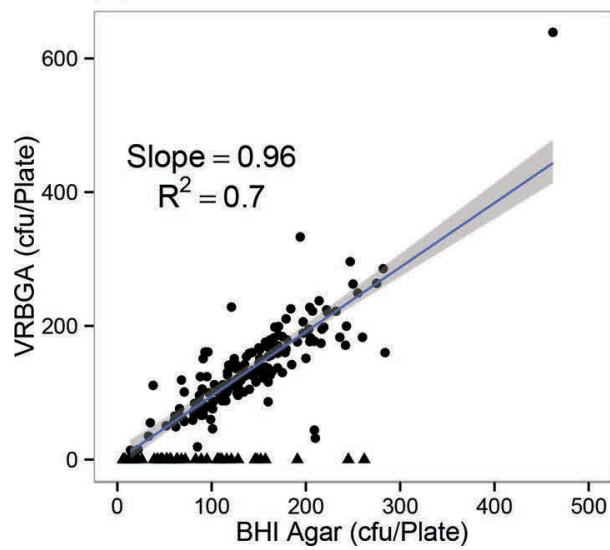

(b.)

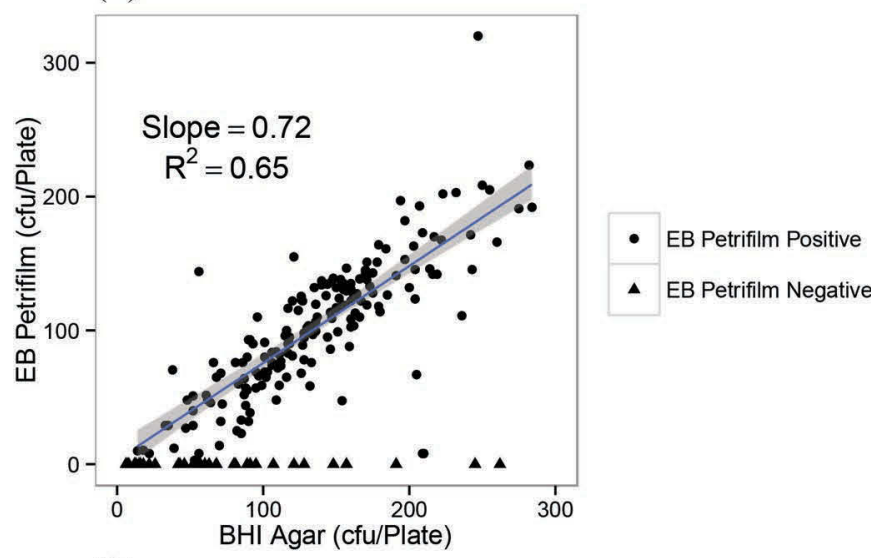

(d.)

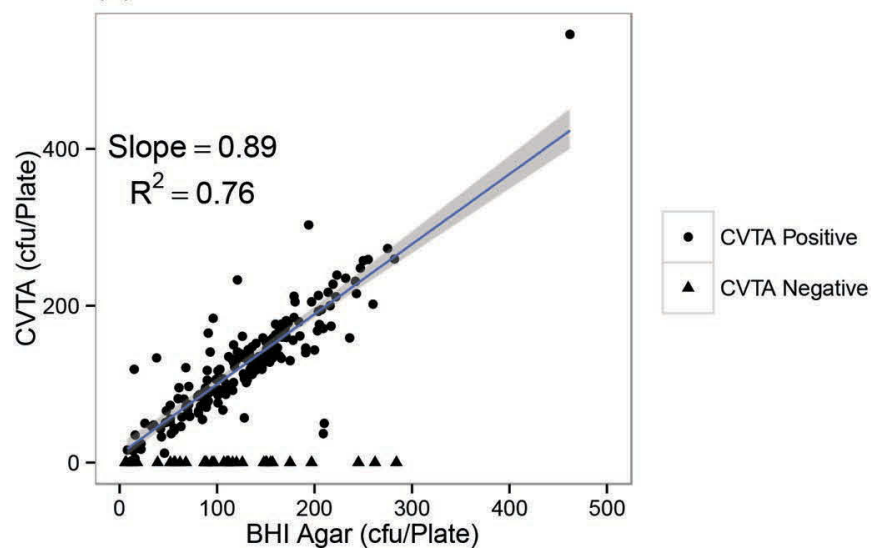

Figure 2. Colony count comparisons between selective/differential detection methods and nonselective brain heart infusion (BHI) agar. Selective/differential detection methods include Coliform Petrifilm (3M, St. Paul, MN) (a), Enterobacteriaceae Petrifilm (EB; b), violet red bile glucose agar (VRBGA; c), and crystal violet tetrazolium agar (CVTA; d). Isolates exhibiting growth on both the selective/differential detection method and BHI are indicated with a $\bullet$, whereas isolates only exhibiting growth on BHI are indicated with a $\mathbf{\Lambda}$. Slope values of $<1$ indicate a lower average recovery of pure cultures grown on selective/differential medium types when compared with the nonselective BHI medium. The $R^{2}$ values indicate the variability of selective/differential medium plate counts and the overall fit of the regression line to the data (an $R^{2}$ of 1 indicates a perfect linear fit). Isolates that showed no growth on a given selective and differential medium (i.e., 0 cfu/plate) were not included in the calculation of slope and $\mathrm{R}^{2}$. Isolates included in the statistical model, but not pictured due to high plate count values, include A5-0095, C40023, C4-0012, and W5-0630 for panels (a) and (b) and A5-0095, C4-0023, and C4-0012 for panels (c) and (d). Color version available online.

medium types when compared with the nonselective BHI medium. The VRBGA demonstrated a recovery most similar to that of BHI as indicated by a slope of 0.96. We observed lower recoveries for CVTA, Coliform Petrifilm, and EB Petrifilm with slopes of 0.89, 0.83, and 0.72 , respectively. Furthermore, we computed $\mathrm{R}^{2}$ values for isolates demonstrating growth on both the selective/differential media and nonselective BHI to evaluate the variability of selective/differential medium plate counts and the overall fit of the regression line to the data (an $\mathrm{R}^{2}$ of 1 indicates a perfect fit). The CVTA and Coliform Petrifilm exhibited the least amount of plate count variability with $\mathrm{R}^{2}$ values of 0.76 . Greater plate count variability was observed for VRBGA and EB Petrifilm with $\mathrm{R}^{2}$ values of 0.70 and 0.65 , respectively.

\section{DISCUSSION}

Despite numerous advances in both processing technology and sanitation practices in the dairy industry over the past 25 yr (Goff and Griffiths, 2006; Marriott and Gravani, 2006), prevention of postpasteurization contamination remains a challenge for many dairy processors. While the occurrence of coliforms in milk has been linked to unhygienic processing conditions and decreased sensory scores of pasteurized milk during product shelf life (Wessels et al., 1989; Martin et al., 2012; Masiello et al., 2016), several studies show noncoliform gram-negative organisms to be the primary culprits of postpasteurization contamination in fluid milk (Craven and Macauley, 1992; Dogan and Boor, 2003). For example, a study evaluating the 
bacterial ecology of HTST milk in the United States found that approximately $75 \%$ of gram-negative organisms isolated from pasteurized milk fall into common noncoliform genera (i.e., Pseudomonas, Acinetobacter, and Flavobacterium; Ranieri and Boor, 2009). Despite the dominating presence of noncoliform gram-negative organisms in pasteurized milk, screening for coliforms remains the standard method by which most dairy processors evaluate the hygienic status of their products and processing environments (Frank and Yousef, 2004). Our data indicate that (1) coliform tests fail to detect several dairy-relevant gram-negative organisms responsible for product spoilage and (2) EB and total gramnegative testing methods detect a large proportion of dairy-associated genera without excluding traditional coliform organisms.

\section{Coliform Tests Fail to Detect a Large Number of Dairy-Relevant Gram-Negative Organisms}

This study highlights a principal shortcoming of coliform tests (i.e., their inability to detect key dairy spoilage organisms commonly isolated from finished dairy products). The $39 \%(82 / 211)$ of isolates that went undetected on Coliform Petrifilm represent a diverse group of organisms from 17 genera, including 10 genera where all isolates went undetected on Coliform Petrifilm. As gram-negative organisms, the isolates that went undetected on Coliform Petrifilm possess the ability to indicate the hygienic status of dairy products or dairy processing environments. The exclusion of noncoliform organisms with regard to postpasteurization contamination screening is emphasized by the fact that most the coliform-negative isolates had been isolated directly from pasteurized milk $(63 \% ; 56 / 82)$. This not only indicates their presence in the US retail milk supply, but also highlights that these organisms would not have been detected during routine coliform testing. Among the group of organisms not detected on Coliform Petrifilm were isolates classified into EB genera with economic and food safety significance to the dairy industry, notably Yersinia and Salmonella and Shigella (Tacket et al., 1984; CDC, 1985; García-Fulgueiras et al., 2001). Additional organisms that are not coliforms and were not detected on Coliform Petrifilm include Pseudomonas and Acinetobacter, genera commonly associated with milk spoilage and sensory defects (Dogan and Boor, 2003; Hantsis-Zacharov and Halpern, 2007). By solely screening for coliforms, processors thus limit their ability to detect and correct instances of postpasteurization contamination.

\section{Enterobacteriaceae and Total Gram-Negative Tests Detect a Large Proportion of Dairy-Associated Genera}

Our results demonstrate that testing for EB offers a more comprehensive indicator for the hygienic status of dairy products and processing environments when compared with coliform organisms. By screening for EB, up to $100 \%$ of coliform organisms are detected along with other dairy-related EB genera that typically lack the ability to ferment lactose (e.g., Yersinia and Salmonella; Imhoff, 2005). Hence, replacement of coliform with most EB tests will continue to detect coliforms, but will allow for improved detection of the organisms whose presence indicates the same type of hygiene issues identified by coliform testing. When considering sensitivity, convenience, and ease of differentiating between positive and negative test results, EB Petrifilm offers distinct advantages over other EB detection methods. Our results were similar to those found by Silbernagel and Lindberg (2002) in that recovery rates of EB isolates on EB Petrifilm were over $95 \%$ and exceeded those of the VRBGA standard. Additionally, a false positive rate of $16 \%$ was observed for EB Petrifilm in the Silbernagel and Lindberg (2002) study compared with a false positive rate of $11 \%$ observed here. By also detecting $100 \%$ of isolates that tested coliform positive, EB Petrifilm offers a more complete screening of potential dairy-related indicator organisms without excluding the conventionally used coliform group. The VRBGA proved to be sensitive and specific in the detection of EB organisms, though the additional space and materials needed for this method make it less convenient. Our results agree with past studies in that certain species within the Aeromonas genus have the ability to ferment glucose and thus may test as false positives when plated on EB selective and differential media (Abbott et al., 2003; Erdem et al., 2011). Similarly, previous studies demonstrate the ability of select Acinetobacter isolates to utilize glucose with acid production, thus potentially testing positive when using EB detection methods (Constantiniu et al., 2004). Despite this, the high specificities of EB testing methods evaluated in this study validate the ability of the tests to correctly identify the vast majority of true negatives.

With time to result proving to be a driving force for the development of alternative indicator organism tests, rapid detection methods (e.g., the D-Count) can have distinct advantages over the traditional medium types. Where traditional EB detection methods such as EB Petrifilm and VRBGA require a 24-h incubation period before obtaining results, the D-Count provides results in approximately $13 \mathrm{~h}$ through a selective en- 
richment step and flow cytometry. Though the 13-h incubation period of the D-Count led to a reduced endresult detection time, the test did have the lowest sensitivity of the EB detection methods assessed. However, optimization of the cut-off threshold for the D-Count may further improve its sensitivity and specificity for the use of testing on various food matrices, but will require additional data. The $\mathrm{D}$-Count was also unique among the EB detection methods in that we observed no instances of false positive test results at a cut-off of 100 counts/mL. Our data thus support the potential for development of more rapid tests for EB, which may further improve adoption of EB as dairy hygiene indicators in countries that currently prefer coliform tests.

Screening for total gram-negative organisms allows for detection of an even broader range of organisms with the potential to indicate the hygienic status of dairy products and processing environments when compared with the EB and coliform groups. In this study, we included Acinetobacter, Aeromonas, Brevundimonas, Flavobacterium, Pseudomonas, and Vibrio to represent dairy-related gram-negative organisms falling outside of the EB and coliform groups. These organisms are largely undetected when using coliform or EB testing methods and are predominant dairy spoilage organisms whose presence indicates hygiene issues (Ternström et al., 1993; Dogan and Boor, 2003; Ranieri and Boor, 2009).

As a selective medium for total gram-negative organisms, we evaluated CVTA for its ability to detect all coliform, EB, and non-EB gram-negative isolates. While most isolates exhibited typical growth and detection on CVTA, several organisms exhibited atypical growth or no growth at all when plated on CVTA $(18 \% ; 37 / 211)$. This led to CVTA having the lowest sensitivity (relative to its target organisms, i.e., all gram-negative organisms for CVTA) of the 5 indicator organism testing methods analyzed. Notably, approximately half of the non-EB, gram-negative isolates did not exhibit expected growth characteristics on CVTA. This demonstrates the need for a more sensitive total gram-negative testing method. Despite the lack of sensitivity, screening for total gram-negative organisms using CVTA detects a more expansive group of potential hygiene indicators than the somewhat limiting coliform group. This is largely due to the inclusion of Pseudomonas in total gram-negative testing. In this study, EB Petrifilm and CVTA detected a similar number of dairy relevant gram-negative organisms (172 and 174, respectively). However, previous studies reveal that Pseudomonas dominate the gram-negative microflora of pasteurized milk and commonly cause dairy product spoilage and sensory defects (Ternström et al., 1993; Ranieri and Boor, 2009). In this study, CVTA detected most the selected Pseudomonas isolates (8/10). On the other hand and as expected, as they are not members of the EB family or coliform group, all Pseudomonas isolates were not detected with EB and coliform testing methods. Hence, a total gram-negative test, such as CVTA, has distinct advantages over EB and coliform testing methods if aiming to detect the maximum number of gram-negative hygiene indicators relevant to pasteurized fluid milk. Although further evaluation of CVTA for exclusivity (i.e., absence of detection of gram-positive bacteria) may be valuable, we have not identified issues with detection of gram-positive bacteria when using CVTA for pasteurized fluid milk testing.

\section{CONCLUSIONS}

Through the testing of 211 dairy-related isolates falling into the coliform, EB, and gram-negative groups, we evaluated standard and alternative hygiene indicator organism tests. Out of the testing methods evaluated, EB Petrifilm was the most sensitive and the DCount was the most specific for the detection of EB. By monitoring for EB or total gram-negative organisms, traditional coliform organisms are detected along with a broad range of dairy-related quality indicators lacking the ability to ferment lactose (yet causing spoilage in dairy products). Although testing for total gramnegative organisms in fluid milk offers advantages to other hygiene indicator groups due to the inclusion of non-EB gram-negative organisms (e.g., Pseudomonas), further studies must evaluate their use as indicators in fermented dairy products. This study highlights the wide range of methods available for the detection of $\mathrm{EB}$ and total gram-negative organisms; however, our data also demonstrate the need for continued development of dairy indicator detection methods that are rapid, reliable, and accurate. For researchers interested in further validation of testing methods, Cornell University and the Food Microbe Tracker database (www.foodmicrobetracker.com) offer access to more than 50,000 bacterial isolates, including the 211 isolates evaluated here.

\section{ACKNOWLEDGMENTS}

This publication was made possible by an unrestricted gift from Chobani Inc. (Norwich, NY), supporting innovative research in dairy quality. We thank them for their generous contribution and continued support.

\section{REFERENCES}

Abbott, S. L., W. K. W. Cheung, and J. M. Janda. 2003. The genus Aeromonas: Biochemical characteristics, atypical reactions, and phenotypic identification schemes. J. Clin. Microbiol. 41:2348 2357 . 
CDC (Centers for Disease Control and Prevention). 1985. Epidemiologic notes and reports update: Milk-borne salmonellosis-Illinois. MMWR Morb. Mortal. Wkly. Rep. 34:215-216.

Constantiniu, S., A. Romaniuc, L. S. Iancu, R. Filimon, and I. Tarași. 2004. Cultural and biochemical characteristics of Acinetobacter spp. strains isolated from hospital units. J. Prev. Med. 12:35-42.

Craven, H. M., and B. J. Macauley. 1992. Microorganisms in pasteurized milk after refrigerated storage 1. Identification of types. Aust. J. Dairy Technol. 47:38-45.

Dogan, B., and K. J. Boor. 2003. Genetic diversity and spoilage potentials among Pseudomonas spp. isolated from fluid milk products and dairy processing plants. Appl. Environ. Microbiol. 69:130-138.

Erdem, B., E. Kariptas, E. Cil, and K. Isik. 2011. Biochemical identification and numerical taxonomy of Aeromonas spp. isolated from food samples in Turkey. Turk. J. Biol. 35:463-472.

European Communities Regulation. 2010. Commission regulation on microbiological criteria for foodstuffs. Commission regulation (EC) No 365/2010. Official Journal of the European Union. 1-3.

FDA (Food and Drug Administration). 2011. Standards for grade "A" milk and milk products. Pages 27-30 in Grade "A" Pasteurized Milk Ordinance. Standards for grade "A" milk and milk products. US Department of Health and Human Services, Public Health Service, Washington, DC.

Feng, P., S. D. Weagant, M. A. Grant, and W. Burkhardt. 2002. Enumeration of Escherichia coli and the coliform bacteria. Chapter 4 in Bacteriological Analytical Manual. 8th ed. US Food and Drug Administration, Center for Food Safety and Applied Nutrition, College Park, MD. Accessed May 20, 2015. http://www.fda.gov/ Food/FoodScienceResearch/LaboratoryMethods/ucm064948.htm.

Frank, J. F., and A. E. Yousef. 2004. Tests for groups of microorganisms. Pages 227-248 in Standard Methods for the Examination of Dairy Products. 17th ed. M. Wehr, ed. Am. Public Health Assoc., Washington, DC.

García-Fulgueiras, A., S. Sánchez, J. J. Guillén, B. Marsilla, A. Aladueña, and C. Navarro. 2001. A large outbreak of Shigella sonnei gastroenteritis associated with consumption of fresh pasteurised milk cheese. Eur. J. Epidemiol. 17:533-538.

Goff, H. D., and M. W. Griffiths. 2006. Major advances in fresh milk and milk products: Fluid milk products and frozen desserts. J. Dairy Sci. 89:1163-1173.

Gunders, D. 2012. Wasted: How America is losing up to 40 percent of its food from farm to fork to landfill. NRDC Issue Paper 12-06- B, Natural Resources Defense Council, Washington, DC.

Hantsis-Zacharov, E., and M. Halpern. 2007. Culturable psychrotrophic bacterial communities in raw milk and their proteolytic and lipolytic traits. Appl. Environ. Microbiol. 73:7162-7168.

Hayes, W., C. H. White, and M. A. Drake. 2002. Sensory aroma characteristics of milk spoilage by Pseudomonas species. J. Food Sci. 67:448-454.

Huck, J. R., N. H. Woodcock, R. D. Ralyea, and K. J. Boor. 2007. Molecular subtyping and characterization of psychrotolerant endospore-forming bacteria in two New York State fluid milk processing systems. J. Food Prot. 70:2354-2364.
Imhoff, J. 2005. Enterobacteriales. Pages 587-850 in Bergey's Manual of Systematic Bacteriology. D. Brenner, N. Krieg, J. Staley, G. Garrity, D. Boone, P. De Vos, M. Goodfellow, F. Rainey, and K.H. Schleifer, ed. Springer, New York, NY.

Ivy, R. A., J. M. Farber, F. Pagotto, and M. Wiedmann. 2013. International Life Science Institute North America Cronobacter (formerly Enterobacter sakazakii) isolate set. J. Food Prot. 76:40-51.

Marie Yeung, P. S., A. DePaola, C. A. Kaysner, and K. J. Boor. 2003. A PCR assay for specific detection of the pandemic Vibrio parahaemolyticus O3:K6 clone from shellfish. J. Food Sci. 68:1459-1466.

Marriott, N. G., and R. B. Gravani. 2006. Principles of Food Sanitation, 5th ed. Springer, New York, NY.

Martin, N. H., N. R. Carey, S. C. Murphy, M. Wiedmann, and K. J. Boor. 2012. A decade of improvement: New York State fluid milk quality. J. Dairy Sci. 95:7384-7390.

Martin, N. H., S. C. Murphy, R. D. Ralyea, M. Wiedmann, and K. J. Boor. 2011. When cheese gets the blues: Pseudomonas fluorescens as the causative agent of cheese spoilage. J. Dairy Sci. $94: 3176-3183$

Masiello, S. N., N. H. Martin, A. Trmčić, M. Wiedmann, and K. J. Boor. 2016. Identification and characterization of psychrotolerant coliform bacteria isolated from pasteurized fluid milk. J. Dairy Sci. 99:130-140.

Ranieri, M. L., and K. J. Boor. 2009. Short communication: Bacterial ecology of high-temperature, short-time pasteurized milk processed in the United States. J. Dairy Sci. 92:4833-4840.

Silbernagel, K. M., and K. G. Lindberg. 2002. Evaluation of the 3M Petrifilm Enterobacteriaceae count plate method for the enumeration of Enterobacteriaceae in foods. J. Food Prot. 65:1452-1456.

Sørhaug, T., and L. Stepaniak. 1997. Psychrotrophs and their enzymes in milk and dairy products: Quality aspects. Trends Food Sci. Technol. 8:35-41.

Tacket, C. O., J. P. Narain, R. Sattin, J. P. Lofgren, C. Konigsberg Jr., R. C. Rendtorff, A. Rausa, B. R. Davis, and M. L. Cohen. 1984. A multistate outbreak of infections caused by Yersinia enterocolitica transmitted by pasteurized milk. JAMA 251:483-486.

Ternström, A., A. M. Lindberg, and G. Molin. 1993. Classification of the spoilage flora of raw and pasteurized bovine milk, with special reference to Pseudomonas and Bacillus. J. Appl. Bacteriol. 75:25-34.

US Treasury Department. 1914. Bacterial standard for drinking water. Pages 2959-2966 in Public Health Report. Vol. 29. US Treasury Department, Washington, DC.

Van Tassell, J. A., N. H. Martin, S. C. Murphy, M. Wiedmann, K. J. Boor, and R. A. Ivy. 2012. Evaluation of various selective media for the detection of Pseudomonas species in pasteurized milk. J. Dairy Sci. 95:1568-1574.

Vangay, P., E. B. Fugett, Q. Sun, and M. Wiedmann. 2013. Food microbe tracker: A web-based tool for storage and comparison of food-associated microbes. J. Food Prot. 76:283-294.

Wessels, D., P. J. Jooste, and J. F. Mostert. 1989. Psychrotrophic, proteolytic and lipolytic properties of Enterobacteriaceae isolated from milk and dairy products. Int. J. Food Microbiol. 9:79-83. 